\title{
Beta Burr XII OR Five Parameter Beta Lomax Distribution: Remarks and Characterizations
}

\author{
Z. Javanshiri \\ Department of Statistics, Ferdowsi University of Mashhad \\ P. O. Box 91775-1159, Mashhad, Iran \\ zo_ja15@um.ac.ir \\ M. Maadooliat* \\ Department of Mathematics, Statistics and Computer Science \\ Marquette University, Milwaukee, WI 53201-1881, USA \\ mehdi@mscs.mu.edu
}

Received 5 March 2014

Accepted 16 May 2014

\begin{abstract}
The distributions taken up in two recently published papers are compared and certain characterizations of them are presented. These characterizations are based on: $(i)$ a simple relationship between two truncated moments; (ii) truncated moments of certain functions of the $n^{\text {th }}$ order statistic; (iii) truncated moments of certain functions of the random variable.
\end{abstract}

Keywords: Beta Burr XII; Beta Lomax; Characterizations.

\section{Introduction}

In a paper entitled "On Five Parameter Beta Lomax Distribution" by Rajab et al. [published, March, 2014], the authors present the pdf (probability density function) and cdf (cumulative distribution function) of a distribution which they call "Five Parameter Beta Lomax" as follows:

$$
\begin{aligned}
f(x)=f(x ; a, b, \mu, s, k)= & \frac{k}{s B(a, b)}\left[1+\left(\frac{x-\mu}{s}\right)\right]^{-(k b+1)} \times \\
& \left\{1-\left[1+\left(\frac{x-\mu}{s}\right)\right]^{-k}\right\}^{a-1}, x>\mu,
\end{aligned}
$$

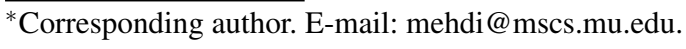


and

$$
\begin{aligned}
F(x)=F(x ; a, b, \mu, s, k)= & \frac{k}{B(a, b)} \sum_{i=0}^{a-1}(-1)^{i}\left(\begin{array}{c}
a-1 \\
i
\end{array}\right) \frac{1}{k b+i k} \times \\
& \left\{1-\left[1+\left(\frac{x-\mu}{s}\right)\right]^{-(k b+i k)}\right\},
\end{aligned}
$$

where $a, b, s, k$ are all positive parameters, $\mu$ is a shift parameter and $B(\alpha, \beta)=$ $\int_{0}^{1} x^{\alpha-1}(1-x)^{\beta-1} \mathrm{~d} x$.

The authors state that their distribution is new and investigate some of its properties. This distribution, however, is a special case of the one introduced by Paranaíba et al. (2011), which they (Paranaíba et al.) call it "The beta Burr XII distribution" with the pdf and cdf given, respectively, by

$$
\begin{aligned}
f(x)=f(x ; a, b, c, s, k)= & \frac{c k}{s^{c} B(a, b)} x^{c-1}\left[1+\left(\frac{x}{s}\right)^{c}\right]^{-(k b+1)} \times \\
& \left\{1-\left[1+\left(\frac{x}{s}\right)^{c}\right]^{-k}\right\}^{a-1}, x>0,
\end{aligned}
$$

and

$$
\begin{aligned}
F(x)=F(x ; a, b, c, s, k) & =I_{1-\left[1+\left(\frac{x}{s}\right)^{c}\right]^{-k}(a, b)} \\
& =\frac{1}{B(a, b)} \int_{0}^{1-\left[1+\left(\frac{x}{s}\right)^{c}\right]^{-k}} \omega^{a-1}(1-\omega)^{b-1} \mathrm{~d} \omega,
\end{aligned}
$$

$x \geq 0$, where $a, b, c, s, k$ are all positive parameters.

Letting $c=1$ and introducing a shift parameter $\mu$, (1.3) reduces to (1.1). Both papers have certain formulas for Reliability and Hazard functions of their respective distributions. Rajab et al. obtain an expression for the $r^{\text {th }}$ moment of the random variable with pdf (1.1) for the special case of $a=1$. This special case, clearly, makes the computations quite easy and straightforward. On the other hand, Paranaíba et al. present $(a)$ several important properties of the random variable with pdf (1.3); $(b)$ derive its moment generating function; $(c)$ discuss other measures, such as: mean deviations, Bonferroni and Lorenz curves; $(d)$ order statistics and moments; $(e)$ maximum likelihood estimation; $(f)$ simulation study; $(g)$ a Bayesian analysis; and $(h)$ an application of their distribution.

In the next section we present certain characterizations of Beta Burr XII (BBXII) distribution. Clearly, these characterizations will hold if the shift parameter $\mu$ is introduced as well.

\section{Characterizations}

In designing a stochastic model for a particular modeling problem, an investigator will be vitally interested to know if their model fits the requirements of a specific underlying probability distribution. To this end, the investigator will rely on the characterizations of the selected distribution. Generally speaking, the problem of characterizing a distribution is an important problem in various 
fields and has recently attracted the attention of many researchers. Consequently, various characterization results have been reported in the literature. These characterizations have been established in many different directions. The present work deals with the characterizations of (BBXII) distribution. These characterizations are based on: $(i)$ a simple relationship between two truncated moments; $(i i)$ truncated moments of certain functions of the $n^{\text {th }}$ order statistic; (iii) truncated moment of certain functions of the random variable.

\subsection{Characterizations based on two truncated moments}

In this subsection we present characterizations of (BBXII) distribution in terms of a simple relationship between two truncated moments. We like to mention here the works of Glänzel $(1987,1990)$, Glänzel and Hamedani (2001) and Hamedani $(2002,2006,2010)$, among others, in this direction. As in Hamedani's works, our characterization results presented here will employ the result due to Glänzel (1987) (Theorem 2.1 below). Following Hamedani, we like to mention that the advantage of this characterization is two fold: it relates the cdf or pdf of a distribution to the solution of a first order differential equation and does not require that the cdf to have a simple format.

Theorem 2.1. Let $(\Omega, \mathscr{F}, \mathbf{P})$ be a given probability space and let $H=[a, b]$ be an interval for some $a<b(a=-\infty, b=\infty$ might as well be allowed $)$. Let $X: \Omega \rightarrow H$ be a continuous random variable with the distribution function $F$ and let $g$ and $h$ be two real functions defined on $H$ such that

$$
\mathbf{E}[g(X) \mid X \geq x]=\mathbf{E}[h(X) \mid X \geq x] \eta(x), \quad x \in H,
$$

is defined with some real function $\eta$. Assume that $g, h \in C^{1}(H), \eta \in C^{2}(H)$ and $F$ is twice continuously differentiable and strictly monotone function on the set $H$. Finally, assume that the equation $h \eta=g$ has no real solution in the interior of $H$. Then $F$ is uniquely determined by the functions $g$, $h$ and $\eta$, particularly

$$
F(x)=\int_{a}^{x} C\left|\frac{\eta^{\prime}(u)}{\eta(u) h(u)-g(u)}\right| \exp (-s(u)) \mathrm{d} u,
$$

where the function $s$ is a solution of the differential equation $s^{\prime}=\frac{\eta^{\prime} h}{\eta h-g}$ and $C$ is a constant, chosen to make $\int_{H} \mathrm{~d} F=1$.

Again following Glänzel and Hamedani (2001), we like to mention that "this kind of characterization based on the ratio of truncated moments is stable in the sense of weak convergence, in particular, let us assume that there is a sequence $\left\{X_{n}\right\}$ of random variables with distribution functions $\left\{F_{n}\right\}$ such that the functions $g_{n}, h_{n}$ and $\eta_{n}(n \in \mathbb{N})$ satisfy the conditions of Theorem 2.1 and let $g_{n} \rightarrow g, h_{n} \rightarrow h$ for some continuously differentiable real functions $g$ and $h$. Let, finally, $X$ be a random variable with distribution $F$. Under the condition that $g_{n}(X)$ and $h_{n}(X)$ are uniformly integrable and the family $\left\{F_{n}\right\}$ is relatively compact, the sequence $X_{n}$ converges to $X$ in distribution if and only if $\eta_{n}$ converges to $\eta$, where

$$
\eta(x)=\frac{E[g(X) \mid X \geq x]}{E[h(X) \mid X \geq x]}
$$

This stability theorem makes sure that the convergence of distribution functions is reflected by corresponding convergence of the functions $g, h$ and $\eta$, respectively. It guarantees, for instance, the 
'convergence' of characterization of the Wald distribution to that of the Lévy-Smirnov distribution if $\alpha \rightarrow \infty$.

A further consequence of the stability property of Theorem 2.1 is the application of this theorem to special tasks in statistical practice such as the estimation of the parameters of discrete distributions. For such purpose, the functions $g, h$ and, specially, $\eta$ should be as simple as possible. Since the function triplet is not uniquely determined it is often possible to choose $\eta$ as a linear function. Therefore, it is worth analyzing some special cases which helps to find new characterizations reflecting the relationship between individual continuous univariate distributions and appropriate in other areas of statistics."

Proposition 2.1. Let $X: \Omega \rightarrow(0, \infty)$ be a continuous random variable and let $h(x)=$ $\left\{1-\left[1+\left(\frac{x}{s}\right)^{c}\right]^{-k}\right\}^{1-a}\left[1+\left(\frac{x}{s}\right)^{c}\right]^{-k b}$ and $g(x)=\left\{1-\left[1+\left(\frac{x}{s}\right)^{c}\right]^{-k}\right\}^{1-a}$, for $x \in(0, \infty)$. The pdf of $X$ is (1.3) if and only if the function $\eta$ defined in Theorem 2.1 has the form

$$
\eta(x)=2\left[1+\left(\frac{x}{s}\right)^{c}\right]^{k b} \text { for } x>0 .
$$

Proof. Let $X$ have density (1.3), then

$$
(1-F(x)) \mathbf{E}[h(X) \mid X \geq x]=\frac{1}{2 b B(\alpha, \beta)}\left[1+\left(\frac{x}{s}\right)^{c}\right]^{-2 k b}, \quad x>0,
$$

and

$$
(1-F(x)) \mathbf{E}[g(X) \mid X \geq x]=\frac{1}{b B(\alpha, \beta)}\left[1+\left(\frac{x}{s}\right)^{c}\right]^{-k b}, \quad x>0
$$

and finally

$$
\eta(x) h(x)-g(x)=\left\{1-\left[1+\left(\frac{x}{s}\right)^{c}\right]^{-k}\right\}^{1-a}>0 \text { for } x>0 .
$$

Conversely, if $\eta$ is given as above, then

$$
s^{\prime}(x)=\frac{\eta^{\prime}(x) h(x)}{\eta(x) h(x)-g(x)}=\frac{2 b c k}{s^{c}} x^{c-1}\left[1+\left(\frac{x}{s}\right)^{c}\right]^{-1}, \quad x>0
$$

and hence

$$
s(x)=\ln \left\{\left[1+\left(\frac{x}{s}\right)^{c}\right]^{2 b k}\right\}, \quad x>0 .
$$

Now, in view of Theorem 2.1, $X$ has density (1.3).

Corollary 2.1. Let $X: \Omega \rightarrow(0, \infty)$ be a continuous random variable and let $h(x)$ be as in Proposition 2.1. The pdf of $X$ is (1.3) if and only if there exist functions $g$ and $\eta$ defined in Theorem 2.1 
satisfying the differential equation

$$
\frac{\eta^{\prime}(x) h(x)}{\eta(x) h(x)-g(x)}=\frac{2 b c k}{s^{c}} x^{c-1}\left[1+\left(\frac{x}{s}\right)^{c}\right]^{-1}, x>0 .
$$

Remark 2.1. (a) The general solution of the differential equation in Corollary 2.1 is

$$
\begin{array}{r}
\eta(x)=\left[1+\left(\frac{x}{s}\right)^{c}\right]^{2 b k}\left[-\int g(x) \frac{2 b c k}{s^{c}} x^{c-1}\left[1+\left(\frac{x}{s}\right)^{c}\right]^{-b k-1} \times\right. \\
\left.\left\{1-\left[1+\left(\frac{x}{s}\right)^{c}\right]^{-k}\right\}^{a-1} \mathrm{~d} x+D\right],
\end{array}
$$

for $x>0$, where $D$ is a constant. One set of appropriate functions is given in Proposition 2.1 with $D=0$.

(b) Clearly there are other triplets of functions $(h, g, \eta)$ satisfying the conditions of Theorem 2.1. We presented one such triplet in Proposition 2.1.

\subsection{Characterizations based on truncated moment of certain functions of the $n^{\text {th }}$ order statistic}

We present here a characterization of (BBXII) distribution based on some functions of the $n^{\text {th }}$ order statistics. Our characterizations will be a consequence of the following proposition, which is similar to the one appeared in Hamedani (2010).

Proposition 2.2. Let $X: \Omega \rightarrow(0, \infty)$ be a continuous random variable with cdf $F$. Let $\psi(x)$ and $q(x)$ be two differentiable functions on $(0, \infty)$ such that $\lim _{x \rightarrow 0} \psi(x)[F(x)]^{n}=0$ and $\int_{0}^{\infty} \frac{q^{\prime}(t)}{[\psi(t)-q(t)]} \mathrm{d} t=\infty$. Then

$$
E\left[\psi\left(X_{n: n}\right) \mid X_{n: n}<t\right]=q(t), t>0,
$$

implies

$$
F(x)=\exp \left\{-\int_{x}^{\infty} \frac{q^{\prime}(t)}{n[\psi(t)-q(t)]} \mathrm{d} t\right\}, \quad x \geq 0
$$

Taking, e.g., $\psi(x)=2\left\{\int_{0}^{1-\left[1+\left(\frac{x}{s}\right)^{c}\right]^{-k}} \omega^{a-1}(1-\omega)^{b-1} \mathrm{~d} \omega\right\}^{n}$ and $q(x)=\frac{1}{2} \psi(x)$, (2.2) will result in (1.4). Clearly, there are other choices for these functions as well.

Remark 2.2. We like to point out that Proposition 2.2 holds true (with of course appropriate modifications) if we replace $X_{n: n}$ with the base random variable $X$.

\subsection{Characterization based on single truncated moment of certain function of the random variable}

In this subsection we employ a single function $\psi_{1}$ of $X$ and characterize the distribution of $X$ in terms of the truncated moment of $\psi_{1}(X)$. The following proposition is similar to the one which has 
already appeared in a Technical Report (Hamedani, 2013), so we will just state it here which can be used to characterize (BBXII) distribution.

Proposition 2.3. Let $X: \Omega \rightarrow(0, \infty)$ be a continuous random variable with cdf $F$. Let $\psi_{1}(x)$ be a differentiable function on $(0, \infty)$ with $\lim _{x \rightarrow \infty} \psi_{1}(x)=1$. Then for $\delta_{1} \neq 1$,

$$
E\left[\psi_{1}(X) \mid X<x\right]=\delta_{1} \psi_{1}(x), \quad x>0,
$$

if and only if

$$
\psi_{1}(x)=(F(x))^{\frac{1}{\delta_{1}}-1} . \quad x \geq 0 .
$$

Remark 2.3. For a suitable choice of $\psi_{1}(x)$, Proposition 2.3 provides a characterization of (BBXII) distribution.

\section{Acknowledgments}

The authors would like to thank an anonymous referee for their comments. We are specially grateful to the Editor, Professor Hamedani, for his suggestions regarding the important references, his guidance in the presentation of the results and for allowing us to quote a few statements from his work.

\section{References}

[1] Glänzel, W., A characterization theorem based on truncated moments and its application to some distribution families, Mathematical Statistics and Probability Theory (Bad Tatzmannsdorf, 1986), Vol. B, Reidel, Dordrecht, (1987) 75-84.

[2] Glänzel, W., Some consequences of a characterization theorem based on truncated moments, Statistics, 21 (1990) 613-618.

[3] Glänzel, W. and Hamedani, G.G., Characterizations of univariate continuous distributions, Studia Sci. Math. Hungar, 37 (2001) 83-118.

[4] Hamedani, G.G., Characterizations of univariate continuous distributions. II, Studia Sci. Math. Hungar., 39 (2002) 407-424.

[5] Hamedani, G.G., Characterizations of univariate continuous distributions. III, Studia Sci. Math. Hungar, 43 (2006) 361-385.

[6] Hamedani, G.G., Characterizations of continuous univariate distributions based on the truncated moments of functions of order statistics, Studia Sci. Math. Hungar, 47 (2010) 462-484.

[7] Hamedani, G.G., On certain generalized gamma convolution distributions II, Technical Report No. 484, MSCS, Marquette University, (2013).

[8] Paranaíba, P.F., Ortega, E.M.M., Cordeiro, G.M. and Pescim, R.R., The beta Burr XII distribution with application to lifetime data, Computational Statistics and Data Analysis, 55 (2011) 1118-36.

[9] Rajab, M., Aleem, M., Nawaz, T. and Daniyal, M., On five parameter beta Lomax distribution, J. of Statistics, 20 (2013) 102-118. 